Pacific Journal of Mathematic 


\title{
MULTIPLE SERIES ROGERS-RAMANUJAN TYPE IDENTITIES
}

\author{
GEORGE E. ANDREWS
}

\begin{abstract}
It is shown how each of the classical identities of Rogers-Ramanujan type can be embedded in an infinite family of multiple series identities. The method of construction is applied to four of L. J. Rogers' elegant series related to the quintuple product identity. Other applications are also presented.
\end{abstract}

1. Introduction. The Rogers-Ramanujan identities [6; Ch. 7] are given analytically as follows:

$$
\begin{aligned}
& 1+\sum_{n=1}^{\infty} \frac{q^{n^{2}}}{(1-q)\left(1-q^{2}\right) \cdots\left(1-q^{n}\right)} \\
&=\prod_{n=0}^{\infty} \frac{1}{\left(1-q^{5 n+1}\right)\left(1-q^{5 n+4}\right)}, \\
& 1+\sum_{n=1}^{\infty} \frac{q^{n^{2}+n}}{(1-q)\left(1-q^{2}\right) \cdots\left(1-q^{n}\right)} \\
&=\prod_{n=0}^{\infty} \frac{1}{\left(1-q^{5 n+2}\right)\left(1-q^{5 n+3}\right)} .
\end{aligned}
$$

Numerous authors [18], [19], [16], [12], [13], [22], [23] in the first half of this century found related results connecting $q$-series resembling those in (1.1) and (1.2) with various modular forms and functions. The culmination of their efforts may be found in the two papers of L. J. Slater [22], [23] wherein over 130 such identities are cataloged.

Within the last decade it has been observed that if one extends the $q$-series allowed to multiple series then infinite families of RogersRamanujan type identities can be found [4], [5], [14], [17], [25], [26]. For example [4],

$$
\begin{gathered}
\sum_{n_{k-1} \geq \cdots \geq n_{1} \geq 0} \frac{q^{n_{1}^{2}+n_{2}^{2}+\cdots+n_{k-1}^{2}}}{(q)_{n_{k-1}-n_{k-2}}(q)_{n_{k-2}-n_{k-3}} \cdots(q)_{n_{1}}} \\
=\prod_{\substack{n=1 \\
n \neq 0, \pm k(\bmod 2 k+1)}}^{\infty}\left(1-q^{n}\right)^{-1},
\end{gathered}
$$


where $(q)_{n}=(1-q)\left(1-q^{2}\right) \cdots\left(1-q^{n}\right)$. Such series have opened several new combinatorial vistas related to such identities [8], [14].

However other identities originally found by Rogers have not been fully generalized to infinite families (work in this direction is given in [17]). The following four results of L. J. Rogers (for a history and combinatorial applications see [11]) are perhaps the best examples of “isolated” Rogers-Ramanujan type identities:

$$
\begin{aligned}
1 & +\sum_{n=1}^{\infty} \frac{q^{n^{2}}}{(1-q)\left(1-q^{2}\right) \cdots\left(1-q^{2 n}\right)} \\
& =\prod_{n=0}^{\infty} \frac{1}{\left(1-q^{2 n+1}\right)\left(1-q^{20 n+4}\right)\left(1-q^{20 n+8}\right)} \\
& =\prod_{n=0}^{\infty} \frac{\left(1-q^{10 n+10}\right)\left(1-q^{10 n+2}\right)\left(1-q^{10 n+8}\right)\left(1-q^{20 n+6}\right)\left(1-q^{20 n+14}\right)}{\left(1-q^{n+1}\right)}
\end{aligned}
$$

(1.5) $\sum_{n=0}^{\infty} \frac{q^{n^{2}+2 n}}{(1-q)\left(1-q^{2}\right) \cdots\left(1-q^{2 n+1}\right)}$

$$
\begin{aligned}
& =\prod_{n=0}^{\infty} \frac{1}{\left(1-q^{2 n+1}\right)\left(1-q^{20 n+8}\right)\left(1-q^{20 n+12}\right)} \\
& =\prod_{n=0}^{\infty} \frac{\left(1-q^{10 n+10}\right)\left(1-q^{10 n+4}\right)\left(1-q^{10 n+6}\right)\left(1-q^{20 n+2}\right)\left(1-q^{20 n+18}\right)}{\left(1-q^{n+1}\right)}
\end{aligned}
$$

(1.6) $\begin{aligned} \sum_{n=0}^{\infty} & \frac{q^{n^{2}+n}}{(1-q)\left(1-q^{2}\right) \cdots\left(1-q^{2 n}\right)}=\prod_{\substack{n \neq 0, \pm 1, \pm 8, \pm 9,10(\bmod 20) \\ n=0}}^{\infty}\left(1-q^{n}\right)^{-1} \\ & =\prod_{n=0}^{\infty} \frac{\left(1-q^{20 n+10}\right)\left(1-q^{10 n+1}\right)\left(1-q^{10 n+9}\right)\left(1-q^{20 n+8}\right)\left(1-q^{20 n+12}\right)}{\left(1-q^{n+1}\right)}\end{aligned}$

$$
\text { (1.7) } \begin{aligned}
\sum_{n=0}^{\infty} & \frac{q^{n^{2}+n}}{(1-q)\left(1-q^{2}\right) \cdots\left(1-q^{2 n+1}\right)}=\prod_{\substack{n \neq 0, \pm 3, \pm 4, \pm 7,10(\bmod 20) \\
n \neq 0}}^{\infty}\left(1-q^{n}\right)^{-1} \\
& =\prod_{n=0}^{\infty} \frac{\left(1-q^{10 n+10}\right)\left(1-q^{10 n+3}\right)\left(1-q^{10 n+7}\right)\left(1-q^{20 n+16}\right)\left(1-q^{20 n+4}\right)}{\left(1-q^{n+1}\right)} .
\end{aligned}
$$

One reason these four are considered noteworthy is because of the appearance of the Quintuple Product Identity [3; p. 466] in each second line. This is especially intriguing in light of the fact that all previous infinite family extensions [4], [5], [14] have involved only the Triple Product of Jacobi [6; p. 21]. 
The object of this paper is to show that all of the 130 identities given in Slater's compendium [21] can be embedded in infinite families of results. In particular, identities (1.4)-(1.7) can be generalized to infinite families. For example, the generalization of (1.4) is

$$
\begin{aligned}
& \sum_{n_{k} \geq n_{k-1} \geq \cdots \geq n_{1} \geq 0} \frac{q^{n_{1}^{2}+n_{2}^{2}+\cdots+n_{k}^{2}}}{(q)_{n_{k}-n_{k-1}}(q)_{n_{k-1}-n_{k-2}} \cdots(q)_{n_{2}-n_{1}}(q)_{2 n_{1}}} \\
&=\prod_{\substack{n \neq 1 \\
n \neq 0, \pm(k+1)(\bmod 6 k+4) \\
n \neq \pm(4 k+2)(\bmod 12 k+8)}}^{\infty}\left(1-q^{n}\right)^{-1} .
\end{aligned}
$$

In fact the extensions of (1.4)-(1.7) presented in this paper all rely on the Quintuple Product Identity (see (3.5), (3.9), (3.13)).

In $\S 2$, we shall prove Theorem 1 which provides the means for these extensions. In $\S 3$ we prove the generalizations of (1.4)-(1.7). In a very natural way certain questions concerning the "reducibility" of RogersRamanujan type identities arise; some initial observations on this topic are provided in $\S 4$. In $\S 5$ we explore how Theorem 1 can be applied to several identities that seem to fall naturally outside of the domain effectively outlined by L. J. Slater in [22] and [23].

The results we prove are most closely related to S. Milne's striking and elegant multiple series expansion for the general Rogers-Selberg function [17]. Indeed Milne sketches applications using the quintuple product identity [17; pp. 641-642], and presumably the specializations he gives in [17; eq. (3.12) abcd] make his function ${ }_{(a)} \phi_{1,1}(x ; q)$ summable to yield our (1.3), (3.5), (3.9) and (3.13). The primary advantage of our approach is that it allows us to avoid having to sum special cases of ${ }_{(a)} \phi_{1,1}(x ; q)$ and instead lets us quote known summations from Slater's extended tables [22]. Indeed one might say that Milne's work represents the full generalization of Rogers' second proof of (1.1) and (1.2) [20] while the results in this paper fully generalize Rogers' first proof [18].

2. The main theorem. The germ of our work lies in W. N. Bailey's fundamental papers [12], [13] on Rogers-Ramanujan type identities. In particular, the very brief $\S 4$ of Bailey's paper [13] provides the mechanism for producing an infinite family of identities out of one identity. To give Bailey's results we require the following standard notation [24; p. 89]:

$$
(a ; q)_{\infty}=(a)_{\infty}=\prod_{n=0}^{\infty}\left(1-a q^{n}\right)
$$

$$
\begin{aligned}
& (a ; q)_{n}=(a)_{n}=(a ; q)_{\infty} /\left(a q^{n} ; q\right)_{\infty} \\
& \left(=(1-a)(1-a q) \cdots\left(1-a q^{n-1}\right) \text { for } n \text { a nonnegative integer }\right) .
\end{aligned}
$$


The assertion by Bailey in $[13 ; \S 4]$ is:

BAILEY'S LEMMA. If for $n \geq 0$

$$
\beta_{n}=\sum_{r=0}^{n} \frac{\alpha_{r}}{(q)_{n-r}(a q)_{n+r}}
$$

then

$$
\begin{array}{r}
\sum_{n \geq 0}\left(\frac{\left(\rho_{1}\right)_{n}\left(\rho_{2}\right)_{n}\left(a q / \rho_{1} \rho_{2}\right)^{n} \alpha_{n}}{\left(a q / \rho_{1}\right)_{n}\left(a q / \rho_{2}\right)_{n}}\right) \frac{1}{(q)_{N-n}(a q)_{N+n}} \\
=\sum_{n \geq 0} \frac{\left(\rho_{1}\right)_{n}\left(\rho_{2}\right)_{n}\left(a q / \rho_{1} \rho_{2}\right)^{n} \beta_{n}\left(a q / \rho_{1} \rho_{2}\right)_{N-n}}{(q)_{N-n}\left(a q / \rho_{1}\right)_{N}\left(a q / \rho_{2}\right)_{N}} .
\end{array}
$$

To obtain the above result one merely takes the results from $[13 ; \S 4]$ and fills them into the identity in [13; §2]. Finally algebraic simplification provides the above form of the result.

The important observation is that (2.4) is again an instance of (2.3) with now

$$
\alpha_{r}^{\prime}=\frac{\left(\rho_{1}\right)_{r}\left(\rho_{2}\right)_{r}\left(a q / \rho_{1} \rho_{2}\right)^{r} \alpha_{r}}{\left(a q / \rho_{1}\right)_{r}\left(a q / \rho_{2}\right)_{r}}
$$

and

$$
\beta_{N}^{\prime}=\sum_{n \geq 0} \frac{\left(a q / \rho_{1} \rho_{2}\right)_{N-n}\left(\rho_{1}\right)_{n}\left(\rho_{2}\right)_{n}\left(a q / \rho_{1} \rho_{2}\right)^{n} \beta_{n}}{(q)_{N-n}\left(a q / \rho_{1}\right)_{N}\left(a q / \rho_{2}\right)_{N}} .
$$

Thus Bailey's Lemma can be iterated ad infinitum, and this iteration is what is made explicit in the following result.

THEOREM 1. Let the sequences $\alpha_{n}$ and $\beta_{n}$ be related by (2.3), then

$$
\begin{aligned}
& \sum_{n \geq 0} \frac{\left(b_{1}\right)_{n}\left(c_{1}\right)_{n}\left(b_{2}\right)_{n}\left(c_{2}\right)_{n} \cdots\left(b_{k}\right)_{n}\left(c_{k}\right)_{n}\left(q^{-N}\right)_{n}}{\left(a q / b_{1}\right)_{n}\left(a q / c_{1}\right)_{n}\left(a q / b_{2}\right)_{n}\left(a q / c_{2}\right)_{n} \cdots\left(a q / b_{k}\right)_{n}\left(a q / c_{k}\right)_{n}\left(a q^{N+1}\right)_{n}} \\
& \times\left(\frac{a^{k} q^{k+N}}{b_{1} c_{1} \cdots b_{k} c_{k}}\right)^{n} q^{-\left(\frac{n}{2}\right)}(-1)^{n} \alpha_{n} \\
& =\frac{(a q)_{N}\left(a q / b_{k} c_{k}\right)_{N}}{\left(a q / b_{k}\right)_{N}\left(a q / c_{k}\right)_{N}} \sum_{n_{k} \geq n_{k-1} \geq \cdots \geq n_{1} \geq 0} \frac{\left(b_{k}\right)_{n_{k}}\left(c_{k}\right)_{n_{k}} \cdots\left(b_{1}\right)_{n_{1}}\left(c_{1}\right)_{n_{1}}}{(q)_{n_{k}-n_{k-1}}(q)_{n_{k-1}-n_{k-2}} \cdots(q)_{n_{2}-n_{1}}} \\
& \times \frac{\left(q^{-N}\right)_{n_{k}}\left(a q / b_{k-1} c_{k-1}\right)_{n_{k}-n_{k-1}} \cdots\left(a q / b_{1} c_{1}\right)_{n_{2}-n_{1}}}{\left(b_{k} c_{k} q^{-N} / a\right)_{n_{k}}\left(a q / b_{k-1}\right)_{n_{k}}\left(a q / c_{k-1}\right)_{n_{k}} \cdots\left(a q / b_{1}\right)_{n_{2}}\left(a q / c_{1}\right)_{n_{2}}} \\
& \times q^{n_{1}+n_{2}+\cdots+n_{k}} a^{n_{1}+\cdots+n_{k-1}}\left(b_{k-1} c_{k-1}\right)^{-n_{k-1}} \cdots\left(b_{1} c_{1}\right)^{-n_{1}} \beta_{n_{1}} .
\end{aligned}
$$


Proof. We proceed by mathematical induction. When $k=1,(2.7)$ reduces to

$$
\begin{aligned}
& \sum_{n \geq 0} \frac{\left(b_{1}\right)_{n}\left(c_{1}\right)_{n}\left(q^{-N}\right)_{n}}{\left(a q / b_{1}\right)_{n}\left(a q / c_{1}\right)_{n}\left(a q^{N+1}\right)_{n}}\left(\frac{a q^{1+N}}{b_{1} c_{1}}\right)^{n}(-1)^{n} q^{-\left(\begin{array}{l}
n \\
2
\end{array}\right)} \alpha_{n} \\
& \quad=\frac{(a q)_{N}\left(a q / b_{1} c_{1}\right)_{N}}{\left(a q / b_{1}\right)_{N}\left(a q / c_{1}\right)_{N}} \sum_{n_{1} \geq 0} \frac{\left(b_{1}\right)_{n_{1}}\left(c_{1}\right)_{n_{1}}\left(q^{-N}\right)_{n_{1}} q^{n_{1}} \beta_{n_{1}}}{\left(b_{1} c_{1} q^{-N} / a\right)_{n_{1}}}
\end{aligned}
$$

which is (2.4) (with $\rho_{1}=b_{1}, \rho_{2}=c_{1}$ ) multiplied by $(q)_{N}(a q)_{N}$ and simplified. Hence the case $k=1$ is merely Bailey's Lemma.

Now assume the result true up to but not including a particular $k$. Then the case $k-1$ is merely a new instance of (2.3) with

$$
\begin{aligned}
\alpha_{n}^{*}= & \frac{\left(b_{1}\right)_{n}\left(c_{1}\right)_{n} \cdots\left(b_{k-1}\right)_{n}\left(c_{k-1}\right)_{n}}{\left(a q / b_{1}\right)_{n}\left(a q / c_{1}\right)_{n} \cdots\left(a q / b_{k-1}\right)_{n}\left(a q / c_{k-1}\right)_{n}} \\
& \times\left(\frac{a^{k-1} q^{k-1}}{b_{1} c_{1} \cdots b_{k-1} c_{k-1}}\right)^{n} \alpha_{n},
\end{aligned}
$$

(2.10) $\beta_{n}^{*}=\frac{\left(a q / b_{k-1} c_{k-1}\right)_{n}}{(q)_{n}\left(a q / b_{k-1}\right)_{n}\left(a q / c_{k-1}\right)_{n}}$

$$
\begin{aligned}
& \times \sum_{n_{k-1} \geq \cdots \geq n_{1} \geq 0} \frac{\left(b_{k-1}\right)_{n_{k-1}}\left(c_{k-1}\right)_{n_{k-1}} \cdots\left(b_{1}\right)_{n_{1}}\left(c_{1}\right)_{n_{1}}}{(q)_{n_{k-1}-n_{k-2}} \cdots(q)_{n_{2}-n_{1}}} \\
& \times \frac{\left(q^{-n}\right)_{n_{k-1}}\left(a b / b_{k-2} c_{k-2}\right)_{n_{k-1}-n_{k-2}} \cdots\left(a q / b_{1} c_{1}\right)_{n_{2}-n_{1}}}{\left(b_{k-1} c_{k-1} q^{-n} / a\right)_{n_{k-1}}\left(a q / b_{k-2}\right)_{n_{k-1}}\left(a q / c_{k-2}\right)_{n_{k-1}} \cdots\left(a q / b_{1}\right)_{n_{2}}\left(a q / c_{1}\right)_{n_{2}}} \\
& \times q^{n_{1}+n_{2}+\cdots+n_{k-1}} a^{n_{1}+\cdots+n_{k-2}}\left(b_{k-2} c_{k-2}\right)^{-n_{k-2}} \cdots\left(b_{1} c_{1}\right)^{-n_{1}} \beta_{n_{1}} .
\end{aligned}
$$

Hence we may substitute $\alpha_{n}^{*}$ and $\beta_{n}^{*}$ into (2.4) with $\rho_{1}=b_{k}, \rho_{2}=c_{k}$ and then multiply through by $(q)_{N}(a q)_{N}$. The resulting lefthand side of (2.4) is obviously the lefthand side of (2.7). The resulting righthand side is

(2.11) $(q)_{N}(a q)_{N} \sum_{n_{k} \geq 0} \frac{\left(b_{k}\right)_{n_{k}}\left(c_{k}\right)_{n_{k}}\left(a q / b_{k} c_{k}\right)^{n_{k}} \beta_{n_{k}}^{*}\left(a q / b_{k} c_{k}\right)_{N-n_{k}}}{(q)_{N-n_{k}}\left(a q / b_{k}\right)_{N}\left(a q / c_{k}\right)_{N}}$

$$
=\frac{(a q)_{N}\left(a q / b_{k} c_{k}\right)_{N}}{\left(a q / b_{k}\right)_{N}\left(a q / c_{k}\right)_{N}} \sum_{n_{k} \geq 0} \frac{\left(b_{k}\right)_{n_{k}}\left(c_{k}\right)_{n_{k}}\left(q^{-N}\right)_{n_{k}} q^{n_{k}} \beta_{n_{k}}^{*}}{\left(b_{k} c_{k} q^{-N} / a\right)_{n_{k}}}
$$




$$
\begin{aligned}
= & \frac{(a q)_{N}\left(a q / b_{k} c_{k}\right)_{N}}{\left(a q / b_{k}\right)_{N}\left(a q / c_{k}\right)_{N}} \sum_{n_{k} \geq 0} \frac{\left(a q / b_{k-1} c_{k-1}\right)_{n_{k}} q^{n_{k}}}{(q)_{n_{k}}\left(a q / b_{k-1}\right)_{n_{k}}\left(a q / c_{k-1}\right)_{n_{k}}} \\
& \times \sum_{n_{k-1} \geq \cdots \geq n_{1} \geq 0} \frac{\left(b_{k}\right)_{n_{k}}\left(c_{k}\right)_{n_{k}} \cdots\left(b_{1}\right)_{n_{1}}\left(c_{1}\right)_{n_{1}}}{(q)_{n_{k-1}-n_{k-2}} \cdots(q)_{n_{2}-n_{1}}} \\
& \times \frac{\left(q^{-N}\right)_{n_{k}}(q)_{n_{k}}\left(a q / b_{k-1} c_{k-1}\right)_{n_{k}-n_{k-1}}}{\left(b_{k} c_{k} q^{-N} / a\right)_{n_{k}}(q)_{n_{k}-n_{k-1}}\left(a q / b_{k-1} c_{k-1}\right)_{n_{k}}}\left(\frac{a}{b_{k-1} c_{k-1}}\right)^{n_{k-1}} \\
& \times \frac{\left(a q / b_{k-2} c_{k-2}\right)_{n_{k-1}-n_{k-2}} \cdots\left(a q / b_{1} c_{1}\right)_{n_{2}-n_{1}}}{\left(a q / b_{k-2}\right)_{n_{k-1}}\left(a q / c_{k-2}\right)_{n_{k-1}} \cdots\left(a q / b_{1}\right)_{n_{2}}\left(a q / c_{1}\right)_{n_{2}}} \\
& \times \frac{(a q)_{N}\left(a q / b_{k} c_{k}\right)_{N}}{\left(a q / b_{k}\right)_{N}\left(a q / c_{k}\right)_{N}} \quad \sum_{n_{k} \geq n_{k-1} \geq \cdots \geq n_{1} \geq 0} \frac{\left(b_{k}\right)_{n_{k}}\left(c_{k}\right)_{n_{k}} \cdots\left(b_{1}\right)_{n_{1}}\left(c_{1}\right)_{n_{1}}}{(q)_{n_{k}-n_{k-1}} \cdots(q)_{n_{2}-n_{1}}} \\
& \times \frac{\left(q^{n_{1}+N}\right)_{n_{k}}\left(a q / b_{k-1} c_{k-1}\right)_{n_{k}-n_{k-1}} \cdots\left(a q / b_{1} c_{1}\right)_{n_{2}-n_{1}}}{\left(b_{k} c_{k} q^{-N} / a\right)_{n_{k}}\left(a q / b_{k-1}\right)_{n_{k}}\left(a q / c_{k-1}\right)_{n_{k}} \cdots\left(a q / b_{1}\right)_{n_{2}}\left(a q / c_{1}\right)_{n_{2}}} \\
& \times q^{n_{1}+\cdots+n_{k}} a^{n_{1}+\cdots+n_{k-1}}\left(b_{k-1} c_{k-1}\right)^{-n_{k-1}} \cdots\left(b_{1} c_{1}\right)^{-n_{1}} \beta_{n_{1}},
\end{aligned}
$$

which is the desired form of the right hand side of (2.7). Thus Theorem 1 is proved.

While Theorem 1 looks cumbersome it has in fact a number of famous and important specializations. The most important occurs for

$$
\begin{gathered}
\alpha_{n}=\frac{\left(1-a q^{2 n}\right)(a)_{n}(-1)^{n} q^{\left(\begin{array}{c}
n \\
2
\end{array}\right)}}{(1-a)(q)_{n}}, \\
\beta_{n}=\delta_{n, 0}=\left\{\begin{array}{lll}
1 & \text { if } & n=0, \\
0 & & n>0 .
\end{array}\right.
\end{gathered}
$$

The fact that $\alpha_{n}$ and $\beta_{n}$ satisfy (2.3) is immediate from the following result (with $M=n$ ) which is easily proved by mathematical induction on $M$.

$$
\sum_{j=0}^{M} \frac{\left(1-a q^{2 J}\right)\left(q^{-n}\right)_{j}(a)_{j} q^{n J}}{(1-a)\left(a q^{n+1}\right)_{j}(q)_{j}}=\frac{(a q)_{M} q^{n M}\left(q^{1-n}\right)_{M}}{(q)_{M}\left(a q^{n+1}\right)_{M}}
$$

a result derived originally by R. P. Agarwal [1; p. 444, final equation].

The case $k=1$ of Theorem 1 with $\alpha_{n}$ given by (2.12) and $\beta_{n}$ given by (2.13) is the terminating case of the limiting form of Jackson's theorem [24; p. 96]. The case $k=2$ is Watson's celebrated $q$-analog of Whipple's Theorem [28] which implies (among many other results) the RogersRamanujan identities (equations (1.1) and (1.2)). 
3. $q$-Series extensions of (1.3)-(1.7). We have chosen four identities from among Slater's list of 130 . However it should be emphasized that Slater derives all 130 identities from $\left(\alpha_{n}, \beta_{n}\right)$ pairs applied to (2.4) with $N \rightarrow \infty$. We may therefore derive for each $\left(\alpha_{n}, \beta_{n}\right)$ pair given by Slater in [22] an infinite family of identities corresponding to $k=1,2,3, \ldots$ in Theorem 1. We have chosen to generalize (1.4)-(1.7) as striking examples of how this works. To simplify our proceedings we consider a special limiting case of Theorem 1 .

THEOREM 2. Let $\alpha_{n}$ and $\beta_{n}$ be related by (2.3). Then

$$
\begin{aligned}
& \frac{1}{(a q)_{\infty}} \sum_{n \geq 0} q^{k n^{2}} a^{k n} \alpha_{n} \\
& \quad=\sum_{n_{k} \geq n_{k-1} \geq \cdots \geq n_{1} \geq 0} \frac{a^{n_{1}+\cdots+n_{k}} q^{n_{1}^{2}+n_{2}^{2}+\cdots+n_{k}^{2}} \beta_{n_{1}}}{(q)_{n_{k}-n_{k-1}}(q)_{n_{k-1}-n_{k-2}} \cdots(q)_{n_{2}-n_{1}}}
\end{aligned}
$$

Proof. Let $N, b_{1}, b_{2}, \ldots, b_{k}, c_{1}, c_{2}, \ldots, c_{k}$ all tend to infinity in Theorem 1.

THEOREM 3. Identity (1.8) is valid.

REMARK. All we need do for this result and the remaining results in this section is to choose the appropriate $\left(\alpha_{n}, \beta_{n}\right)$ pair given by Slater [22] and fill it in to (3.1).

Proof. Since (1.4) appears as identity (98) in Slater [23] we see that the requisite $\left(\alpha_{n}, \beta_{n}\right)$ pair in [22] is given by her $A(1)$, i.e.

$$
\alpha_{m}= \begin{cases}-q^{6 n^{2}-5 n+1}, & m=3 n-1>0, \\ q^{6 n^{2}-n}+q^{6 n^{2}+n}, & m=3 n>0, \\ -q^{6 n^{2}+5 n+1}, & m=3 n+1>0, \\ 1, & m=0,\end{cases}
$$

$$
\beta_{n}=1 /(q)_{2 n} \text {, }
$$


and furthermore $a=1$ (Slater's $x$ is our $a q^{-1}$ ). Hence by Theorem 2 (with $a=1$ ),

$$
\begin{aligned}
& \sum_{n_{k} \geq \cdots \geq n_{1} \geq 0} \frac{q^{n_{1}^{2}+n_{2}^{2}+\cdots+n_{k}^{2}}}{(q)_{n_{k}-n_{k-1}}(q)_{n_{k-1}-n_{k-2}} \cdots(q)_{n_{2}-n_{1}}(q)_{2 n_{1}}} \\
& =\frac{1}{(q)_{\infty}}\left(\sum_{n=1}^{\infty} q^{k(3 n-1)^{2}}\left(-q^{6 n^{2}-5 n+1}\right)+1+\sum_{n=1}^{\infty} q^{k(3 n)^{2}}\left(q^{6 n^{2}-n}+q^{6 n^{2}+n}\right)\right. \\
& \left.\quad+\sum_{n=0}^{\infty} q^{k(3 n+1)^{2}}\left(-q^{6 n^{2}+5 n+1}\right)\right) \\
& =\frac{1}{(q)_{\infty}} \sum_{n=-\infty}^{\infty} q^{(9 k+6) n^{2}+n}-q^{k+1} \sum_{n=-\infty}^{\infty} q^{(9 k+6) n^{2}+(6 k+5) n} \\
& =\frac{1}{(q)_{\infty}} \prod_{n=0}^{\infty}\left(1-q^{(6 k+4)(n+1)}\right)\left(1-q^{k+1+(6 k+4) n}\right)\left(1-q^{5 k+3+(6 k+4) n}\right) \\
& \quad \times\left(1-q^{4 k+2+(12 k+8) n}\right)\left(1-q^{8 k+6+(12 k+8) n}\right) \\
& \quad(\text { by }[6 ; \text { p. 21, Th. 2.8]) } \\
& \quad \prod_{\substack{n \neq 0, \pm(k+1)(\bmod 6 k+4) \\
n \neq \pm(4 k+2)(\bmod 12 k+k)}}^{\infty}\left(1-q^{n}\right)^{-1},
\end{aligned}
$$

as desired.

\section{THEOREM 4.}

$$
\begin{aligned}
& \sum_{n_{k} \geq n_{k-1} \geq \cdots \geq n_{1} \geq 0} \frac{q^{n_{1}^{2}+n_{2}^{2}+\cdots+n_{k}^{2}+2 n_{1}+n_{2}+\cdots+n_{k}}}{(q)_{n_{k}-n_{k-1}}(q)_{n_{k-1}-n_{k-2}} \cdots(q)_{n_{2}-n_{1}}(q)_{2 n_{1}+1}} \\
&=\prod_{\substack{n \neq 0, \pm(2 k+2)(\bmod 6 k+4) \\
n \neq \pm 2 k(\bmod 12 k+8)}}^{\infty}\left(1-q^{n}\right)^{-1} .
\end{aligned}
$$

Proof. This result reduces to (1.5) when $k=1$, and since (1.5) appears as identity (96) in Slater [23] we see that the requisite $\left(\alpha_{n}, \beta_{n}\right)$ pair in [22] is given by her $A(4)$, i.e.

$$
\alpha_{m}= \begin{cases}q^{6 n^{2}-4 n}, & m=3 n-1>0, \\ q^{6 n^{2}+4 n}, & m=3 n>0, \\ -q^{6 n^{2}+8 n+2}-q^{6 n^{2}+4 n}, & m=3 n+1>0 \\ 1, & m=0,\end{cases}
$$




$$
\beta_{n}=q^{n} /\left(q^{2} ; q\right)_{2 n}
$$

and furthermore $a=q$.

Hence by Theorem 2, with $a=q$ and both sides multiplied by $(1-q)^{-1}$

$$
\begin{aligned}
& \sum_{n_{k} \geq n_{k-1} \geq \cdots \geq n_{1} \geq 0} \frac{q^{n_{1}^{2}+n_{2}^{2}+\cdots+n_{k}^{2}+2 n_{1}+n_{2}+\cdots+n_{k}}}{(q)_{n_{k}-n_{k-1}}(q)_{n_{k-1}-n_{k-2}} \cdots(q)_{n_{2}-n_{1}}(q)_{2 n_{1}+1}} \\
& =\frac{1}{(q)_{\infty}}\left(\sum_{n=1}^{\infty} q^{k(3 n-1)^{2}+k(3 n-1)}\left(q^{6 n^{2}-4 n}\right)+1+\sum_{n=1}^{\infty} q^{k(3 n)^{2}+3 k n}\left(q^{6 n^{2}+4 n}\right)\right. \\
& \left.+\sum_{n=0}^{\infty} q^{k(3 n+1)^{2}+k(3 n+1)}\left(-q^{6 n^{2}+8 n+2}-q^{6 n^{2}+4 n}\right)\right) \\
& =\frac{1}{(q)_{\infty}}\left(\sum_{n=-\infty}^{\infty} q^{(9 k+6) n^{2}+(3 k+4) n}-q^{2 k} \sum_{n=-\infty}^{\infty} q^{(9 k+6) n^{2}+(9 k+4) n}\right) \\
& =\frac{1}{(q)_{\infty}}\left(\sum_{n=-\infty}^{\infty} q^{(9 k+6) n^{2}+(3 k+4) n}-q^{2 k+2} \sum_{n=-\infty}^{\infty} q^{(9 k+6) n^{2}+(9 k+8) n}\right) \\
& \text { (replacing } n \text { by }-n-1 \text { in the second sum) } \\
& =\frac{1}{(q)_{\infty}} \prod_{n=0}^{\infty}\left(1-q^{(6 k+4)(n+1)}\right)\left(1-q^{2 k+2+(6 k+4) n}\right)\left(1-q^{4 k+2+(6 k+4) n}\right) \\
& \times\left(1-q^{10 k+8+(12 k+8) n}\right)\left(1-q^{2 k+(12 k+8) n}\right) \\
& \text { (by }[6 ; \text { p. 21, Th. 2.8]) } \\
& =\prod_{\substack{n=1 \\
n \neq 0, \pm(2 k+2)(\bmod 6 k+4) \\
n \neq \pm 2 k(\bmod 12 k+8)}}^{\infty}\left(1-q^{n}\right)^{-1},
\end{aligned}
$$

as desired.

\section{THEOREM 5.}

$$
\begin{aligned}
& \sum_{n_{k} \geq n_{k-1} \geq \cdots \geq n_{1} \geq 0} \frac{q^{n_{1}^{2}+n_{2}^{2}+\cdots+n_{k}^{2}+n_{1}}}{(q)_{n_{k}-n_{k-1}}(q)_{n_{k-1}-n_{k-2}} \cdots(q)_{n_{2}-n_{1}}(q)_{2 n_{1}}} \\
&=\prod_{\substack{n \neq 1 \\
n \neq 0, \pm k(\bmod 6 k+4) \\
n \neq \pm(4 k+4)(\bmod 12 k+8)}}^{\infty}\left(1-q^{n}\right)^{-1}
\end{aligned}
$$

Proof. This result reduces to (1.6) when $k=1$, and since (1.6) appears as identity (99) in Slater [23] we see that the requisite $\left(\alpha_{n}, \beta_{n}\right)$ pair in [22] 
is given by her $A(3)$, i.e.

$$
\alpha_{m}= \begin{cases}-q^{6 n^{2}-2 n}, & m=3 n-1>0, \\ q^{6 n^{2}-2 n}+q^{6 n^{2}+2 n}, & m=3 n>0, \\ -q^{6 n^{2}+2 n}, & m=3 n+1>0 \\ 1, & m=0,\end{cases}
$$

$$
\beta_{n}=q^{n} /(q)_{2 n},
$$

and furthermore $a=1$.

Hence by Theorem 2, with $a=1$

$$
\begin{aligned}
& \sum_{n_{k} \geq n_{k-1} \geq \cdots \geq n_{1} \geq 0} \frac{q^{n_{1}^{2}+n_{2}^{2}+\cdots+n_{k}^{2}+n_{1}}}{(q)_{n_{k}-n_{k-1}}(q)_{n_{k-1}-n_{k-2}} \cdots(q)_{n_{2}-n_{1}}(q)_{2 n_{1}}} \\
& =\frac{1}{(q)_{\infty}}\left(\sum_{n=1}^{\infty} q^{k(3 n-1)^{2}}\left(-q^{6 n^{2}-2 n}\right)+1\right. \\
& \left.\quad+\sum_{n=1}^{\infty} q^{k(3 n)^{2}}\left(q^{6 n^{2}-2 n}+q^{6 n^{2}+2 n}\right)+\sum_{n=0}^{\infty} q^{k(3 n+1)^{2}}\left(-q^{6 n^{2}+2 n}\right)\right) \\
& =\frac{1}{(q)_{\infty}}\left(\sum_{n=-\infty}^{\infty} q^{(9 k+6) n^{2}-2 n}-q^{k} \sum_{n=-\infty}^{\infty} q^{(i k+6) n^{2}+(6 k+2) n}\right) \\
& =\frac{1}{(q)_{\infty}} \prod_{n=0}^{\infty}\left(1-q^{(6 k+4)(n+1)}\right)\left(1-q^{k+(6 k+4) n}\right)\left(1-q^{(5 k+4)+(6 k+4) n}\right) \\
& =\quad\left(1-q^{4 k+4+(12 k+8) n}\right)\left(1-q^{8 k+4+(12 k+8) n}\right) \\
& \quad \quad \quad \text { [6; p. 21, Th. 2.8]) } \\
& \prod_{\substack{n \neq 0, \pm k(\bmod 6 k+4) \\
n \neq \pm(4 k+4)(\bmod 12 k+8)}}^{\infty} \quad\left(1-q^{n}\right)^{-1},
\end{aligned}
$$

as desired.

Finally we have

\section{THEOREM 5.}

$$
\begin{gathered}
\sum_{n_{h} \geq n_{k-1} \geq \cdots \geq n_{1} \geq 0} \frac{q^{n_{1}^{2}+n_{2}^{2}+\cdots+n_{k}^{2}+n_{1}+n_{2}+\cdots+n_{k}}}{(q)_{n_{k}-n_{k-1}}(q)_{n_{k-1}-n_{k-2}} \cdots(q)_{n_{2}-n_{1}}(q)_{2 n_{1}+1}} \\
=\prod_{\substack{n \neq 0, \pm \pm(2 k+1)(\bmod 6 k+4) \\
n \neq \pm(2 k+2)(\bmod 12 k+8)}}^{\infty}\left(1-q^{n}\right)^{-1} .
\end{gathered}
$$


Proof. This result reduces to (1.7) when $k=1$, and since (1.7) appears as identity (94) in Slater [23] we see that the requisite $\left(\alpha_{n}, \beta_{n}\right)$ pair in [22] is given by her $A(2)$, i.e.

$$
\alpha_{m}= \begin{cases}q^{6 n^{2}-n}, & m=3 n-1>0, \\ q^{6 n^{2}+n}, & m=3 n \geq 0 \\ -q^{6 n^{2}+5 n+1}-q^{6 n^{2}+7 n+2}, & m=3 n+1>0\end{cases}
$$

$$
\beta_{n}=1 /\left(q^{2} ; q\right)_{2 n},
$$

and here $a=q$.

Hence by Theorem 2 with $a=q$ and both sides multiplied by $(1-q)^{-1}$

$$
\begin{aligned}
& \sum_{n_{k} \geq n_{k-1} \geq \cdots \geq n_{1} \geq 0} \frac{q^{n_{1}^{2}+n_{2}^{2}+\cdots+n_{k}^{2}+n_{1}+n_{2}+\cdots+n_{k}}}{(q)_{n_{k}-n_{k-1}}(q)_{n_{k-1}-n_{k-2}} \cdots(q)_{n_{2}-n_{1}}(q)_{2 n_{1}+1}} \\
& =\frac{1}{(q)_{\infty}}\left(\sum_{n=1}^{\infty} q^{k(3 n-1)^{2}+k(3 n-1)}\left(q^{6 n^{2}-n}\right)+\sum_{n=0}^{\infty} q^{k(3 n)^{2}+k 3 n}\left(q^{6 n^{2}+n}\right)\right. \\
& \left.\quad+\sum_{n=0}^{\infty} q^{k(3 n+1)^{2}+k(3 n+1)}\left(-q^{6 n^{2}+5 n+1}-q^{6 n^{2}+7 n+2}\right)\right) \\
& =\frac{1}{(q)_{\infty}}\left(\sum_{n=-\infty}^{\infty} q^{(9 k+6) n^{2}+3 k n+n}-q^{2 k+1} \sum_{n=-\infty}^{\infty} q^{(9 k+6) n^{2}+(9 k+5) n}\right) \\
& =\frac{1}{(q)_{\infty}} \prod_{n=0}^{\infty}\left(1-q^{(6 k+4)(n+1)}\right)\left(1-q^{2 k+1+(6 k+4) n}\right)\left(1-q^{4 k+3+(6 k+4) n}\right) \\
& =\prod_{\substack{n \neq 0, \pm(2 k+1)(\bmod 6 k+4) \\
n \neq \pm(2 k+2)(\bmod 12 k+8)}}^{\infty} \times\left(1-q^{2 k+2+(12 k+8) n}\right)\left(1-q^{10 k+6+(12 k+8) n}\right)
\end{aligned}
$$

as desired.

As is obvious from the above, each pair $\left(\alpha_{n}, \beta_{n}\right)$ given by Slater in [22] can be inserted in Theorem 2 or Theorem 1 . The four examples we have chosen constitute applications to probably the most elegant results considered by Rogers et al. beyond the original Rogers-Ramanujan identities. 
4. The pairs of sequences $\left(\alpha_{n}, \beta_{n}\right)$. Often in this subject one may be given only one of the sequences $\alpha_{n}$ or $\beta_{n}$. If the $\alpha_{n}$ are given then the $\beta_{n}$ are determined by (2.3). Less well known but still of importance is the "inversion" of (2.3). Namely [7; p. 8, Lemma 3]

$$
\alpha_{n}=\left(1-a q^{2 n}\right) \sum_{j=0}^{n} \frac{(a q)_{n+j-1}(-1)^{n-j} q^{(n-j)}}{(q)_{n-j}} \beta_{j} .
$$

Also of interest is the creation of $\left(\alpha_{n}^{\prime}, \beta_{n}^{\prime}\right)$ given by Bailey's Lemma. Furthermore if $\left(\alpha_{n}^{\prime}, \beta_{n}^{\prime}\right)$ are given then one can immediately determine $\alpha_{n}$ from (2.5), and one can solve for $\beta_{0}, \beta_{1}, \ldots, \beta_{N}$ from the diagonal system of equations (2.6). Thus the operation of forming the sequence of pairs:

$$
\left(\alpha_{n}, \beta_{n}\right) \rightarrow\left(\alpha_{n}^{\prime}, \beta_{n}^{\prime}\right) \rightarrow\left(\alpha_{n}^{\prime \prime}, \beta_{n}^{\prime \prime}\right) \rightarrow
$$

which was codified explicitly in Theorem 1 can, in fact, be extended to the left as well. In actual fact, if we consider $\alpha_{n}=\alpha_{n}^{0}, \beta_{n}=\beta_{n}^{0}$, then we can form

$$
\begin{aligned}
\cdots & \rightarrow\left(\alpha_{n}^{(-2)}, \beta_{n}^{(-2)}\right) \rightarrow\left(\alpha_{n}^{(-1)}, \beta_{n}^{(-1)}\right) \rightarrow\left(\alpha_{n}^{0}, \beta_{n}^{0}\right) \\
& \rightarrow\left(\alpha_{n}^{1}, \beta_{n}^{1}\right) \rightarrow \cdots
\end{aligned}
$$

where each pair is related to the next through instances of (2.5) and (2.6). In the following, we shall refer to a pair of sequences $\left(\alpha_{n}, \beta_{n}\right)$ related by (2.3) to be a Bailey pair. We shall call (4.2) an ordinary Bailey chain, and (4.3) a bilateral Bailey chain. For any given Bailey chain one would like to designate a "simplest" Bailey pair as the "reduced" element of the chain. At the current state of knowledge it does not appear to be fruitful (or perhaps possible) to provide a rigorous definition of "reduced". Suffice it to say that the Bailey pair given by (2.12) and (2.13) are the obvious "reduced" pair in their family.

Another important way to produce new Bailey pairs $\left(\alpha_{n}, \beta_{n}\right)$ from previous ones is through the replacements $a \rightarrow a^{-1}, q \rightarrow q^{-1}$. Thus if $\alpha_{n}=\alpha_{n}(a, q), \beta_{n}=\beta_{n}(a, q)$,

$$
\begin{aligned}
& A_{k}(a, q)=A_{k}=a^{k} q^{k^{2}} \alpha_{k}\left(a^{-1}, q^{-1}\right), \\
& B_{k}(a, q)=B_{k}=a^{-k} q^{-k^{2}-k} \beta_{k}\left(a^{-1}, q^{-1}\right),
\end{aligned}
$$

then the replacement of $a$ by $a^{-1}$ and $q$ by $q^{-1}$ in (2.3) yields

$$
B_{n}=\sum_{r=0}^{n} \frac{A_{r}}{(q)_{n-r}(a q)_{n+r}} .
$$


Thus the substitution $a \rightarrow a^{-1}, q \rightarrow q^{-1}$ yields a new pair satisfying (2.3). We call $\left(A_{n}, B_{n}\right)$ the dual Bailey pair of $\left(\alpha_{n}, \beta_{n}\right)$.

For example, the Bailey pair $\left(\alpha_{n}, \beta_{n}\right)$ given by (2.12) and (2.13) is self-dual. On the other hand Slater's A-Table [22] of eight pairs $\left(\alpha_{n}, \beta_{n}\right)$ consists of the four pairs we utilized in $\S 3$ and the 4 duals of these pairs.

5. New applications. The $q$-series identities that have previously appeared primarily have their genesis in the work of L. J. Rogers [18], [19]. Indeed the majority of Bailey pairs given by Slater in [22] arise, as she notes, from Rogers' pathbreaking papers. In fact, the Bailey pairs used in the classical development of this subject have always taken $\alpha_{n}$ as zero or plus or minus a power of $q$. The $\beta_{n}$ have always been simple finite $q$-products.

Let us start by delving into the work of I. Schur on the RogersRamanujan type identities. Schur in his famous initial paper [21] on this topic provided three polynomial identities that fit the Bailey pair criterion. Namely he proved (where $\left[{ }_{B}^{A}\right]=(q)_{A} /\left((q)_{B}(q)_{A-B}\right)$ and $[x]$ is the greatest integer in $x$ )

$$
\begin{gathered}
\sum_{\lambda=-\infty}^{\infty}(-1)^{\lambda} q^{\lambda(3 \lambda+1) / 2}\left[\left[\frac{n-3 \lambda}{2}\right]\right]=1, \\
\sum_{\lambda=-\infty}^{\infty}(-1)^{\lambda} q^{\lambda(5 \lambda+1) / 2-2 \alpha \lambda}\left[\left[\frac{n-5 \lambda}{2}\right]+\alpha\right] \\
=\sum_{j \geq 0} q^{j^{2}+\alpha j}\left[\begin{array}{c}
n-\alpha-j] \\
j
\end{array}\right],
\end{gathered}
$$

where in (5.2) $\alpha=0$ or 1. Actually (5.2) first appears in [2]; however, as noted there, the essential observations for this identity go back to Schur. From (5.1) and (5.2) one can obtain 12 Bailey pairs: the first 6 come from $n$ even or odd, $\alpha=0$ or 1 . The last 6 are the dual Bailey pairs of the first 6. In fact, Rogers had proved (5.1) in [18] since when $n$ is even (5.1) is Bailey pair $A(1)$ of [22] and when $n$ is odd (5.1) is Bailey pair $A(2)$ of [22]. As mentioned previously, Rogers also had the dual Bailey pairs of $A(1)$ and $A(2)$. Of the remaining eight possible Bailey pairs implied by (5.2), we choose the case $n$ even, $\alpha=0$. In this instance the Bailey pair is

$$
\alpha_{n}= \begin{cases}q^{m(10 m-1)}+q^{m(10 m+1)}, & n=5 m>0, \\ 1, & n=0, \\ -q^{10 m^{2}+11 m+3}, & n=5 m+3>0, \\ -q^{10 m^{2}-11 m+3}, & n=5 m-3>0 .\end{cases}
$$




$$
\beta_{n}=\frac{1}{(q)_{2 n}} \sum_{j=0}^{n} q^{J^{2}}\left[\begin{array}{c}
2 n-j \\
j
\end{array}\right]
$$

where $a=1$.

When this pair is used in conjunction with Theorem 2 , the resulting identity is

$$
\begin{gathered}
\text { (5.5) } \frac{1}{(q)_{\infty}}\left(\prod_{m=0}^{\infty}\left(1-q^{(m+1)(50 k+20)}\right)\left(1+q^{(25 k+9)+(50 k+20) m}\right)\left(1+q^{(25 k+11)+(50 k+20) m}\right)\right. \\
\left.-q^{4 k+2} \prod_{m=0}^{\infty}\left(1-q^{(m+1)(50 k+20)}\right)\left(1+q^{5 k+1+(50 k+20) m}\right)\left(1+q^{45 k+19+(50 k+20) m}\right)\right) \\
\quad=\sum_{n_{k} \geq \cdots \geq n_{1} \geq n_{0} \geq 0} \frac{q^{n_{k}^{2}+\cdot+n_{1}^{2}+n_{0}^{2}}(q)_{2 n_{1}-n_{0}}}{(q)_{n_{k}-n_{k-1}} \cdots(q)_{n_{2}-n_{1}}(q)_{2 n_{1}-2 n_{0}}(q)_{2 n_{1}}(q)_{n_{0}}} .
\end{gathered}
$$

D. Bressoud [15], in a study building on Rogers' work, has found several other new Bailey pairs. As an example, we list

$$
\alpha_{n}= \begin{cases}(-1)^{m}\left(q^{m(5 m+1) / 2}+q^{m(5 m-1) / 2}\right), & n=2 m, \\ 1, & n=0, \\ 0, & n \text { odd },\end{cases}
$$

$$
\beta_{n}=\frac{1}{(q)_{2 n}} \sum_{j=0}^{n}\left[\begin{array}{l}
n \\
j
\end{array}\right] q^{J^{2}}
$$

where $a=1$.

Hence by Theorem 2,

$$
\begin{gathered}
\sum_{n_{k} \geq \cdots \geq n_{1} \geq n_{0} \geq 0} \frac{q^{n_{k}^{2}+\cdots+n_{1}^{2}+n_{0}^{2}}(q)_{n_{1}}}{(q)_{n_{k}-n_{k-1}} \cdots(q)_{n_{2}-n_{1}}(q)_{n_{1}-n_{0}}(q)_{n_{0}}(q)_{2 n_{1}}} \\
=\prod_{\substack{n \neq 1 \\
n \neq 0, \pm(4 k+2)(\bmod 8 k+5)}}^{\infty}\left(1-q^{n}\right)^{-1},
\end{gathered}
$$

which, of course, reduces to (1.1) for $k=0$.

Finally, and most off the beaten track, we shall see that the Wall polynomials [27] from group theory fit into the Bailey pair scheme.

The Wall polynomials were first defined in [27] in order to determine the generating functions for the numbers of conjugacy classes in the symplectic and orthogonal groups over finite fields of characteristic 2 . They are defined by the following recurrences:

$$
X_{2 n+1}=X_{2 n}+q^{2 n+1} X_{2 n-1},
$$


(5.10) $X_{2 n+2}=X_{2 n+1}+q^{n+1}\left(1+q^{n+1}\right)\left(X_{2 n+1}+\left(1-q^{2 n+1}\right) X_{2 n-1}\right)$, where $X_{-1}=X_{-1}(a, b, q)=a$ and $X_{0}=X_{0}(a, b, q)=b$.

Recently it has been shown that $[\mathbf{1 0}]$ among other results

$$
\begin{aligned}
& X_{2 n-1}(1,1, q)=\sum_{j=-\infty}^{\infty} q^{j^{2}}\left[\begin{array}{c}
2 n \\
n+2 j
\end{array}\right], \\
& X_{2 n-1}(0,1, q)=\sum_{j \geq 0} q^{j^{2}+j}\left[\begin{array}{c}
2 n \\
n-2 j-1
\end{array}\right] .
\end{aligned}
$$

Hence $\beta_{n}=X_{2 n-1}(1,1, q) /(q)_{2 n}$ forms a Bailey pair with

$$
\alpha_{n}= \begin{cases}2 q^{J^{2}}, & n=2 j \\ 1, & n=0 \\ 0, & n \text { odd }\end{cases}
$$

and $a=1$.

Thus by Theorem 2

$$
\begin{gathered}
\sum_{n_{k} \geq \cdots \geq n_{1} \geq 0} \frac{q^{n_{k}^{2}+\cdots+n_{1}^{2}} X_{2 n_{1}-1}(1,1, q)}{(q)_{n_{k}-n_{h-1}} \cdots(q)_{n_{2}-n_{1}}(q)_{2 n_{1}}} \\
=\frac{1}{(q)_{\infty}} \sum_{j=-\infty}^{\infty} q^{(4 k+1) \jmath^{2}} .
\end{gathered}
$$

Finally $\beta_{n}=X_{2 n-1}(0,1, q) /(q)_{2 n}$ forms a Bailey pair with

$$
\alpha_{n}= \begin{cases}q^{j^{2}+J}, & n=2 j+1 \\ 0, & n \text { even }\end{cases}
$$

with $a=1$.

Hence by Theorem 2

$$
\begin{gathered}
\sum_{n_{h} \geq \cdots \geq n_{1} \geq 0} \frac{q^{n_{k}^{2}+\cdots+n_{1}^{2}} X_{2 n_{1}-1}(0,1, q)}{(q)_{n_{k}-n_{k-1}} \cdots(q)_{n_{2}-n_{1}}(q)_{2 n_{1}}} \\
=\frac{\sum_{J=0}^{\infty} q^{k(2 J+1)^{2}+J^{2}+j}}{(q)_{\infty}} .
\end{gathered}
$$

6. Conclusion. Our object has not been to add heaps of further Rogers-Ramanujan identities to the already extended lists. Rather it has been to exhibit clearly mechanisms for their construction. The problems 
of additive number theory [8], combinatorics and special function [5], and physics [9] will presumably dictate which Bailey pairs and Bailey chains will be of most interest. Presumably we have here provided the framework for their construction.

\section{REFERENCES}

[1] R. P. Agarwal, On the partial sums of series of hypergeometric type, Proc. Cambridge Phil. Soc., 49 (1953), 441-445.

[2] G. E. Andrews, A polynomial identity which implies the Rogers-Ramanujan identities, Scripta Math., 28 (1970), 297-305.

[3] Applications of basic hypergeometric functions, S.I.A.M. Review, 16 (1974), 441-484.

[4] __ An analytic generalization of the Rogers-Ramanujan identities for odd moduli, Proc. Nat. Acad. Sci. U.S.A., 71 (1974), 4082-4085.

[5] _ Problems and Prospects for Basic Hypergeometric Functions, The Theory and Applications of Special Functions, (R. Askey, ed.), Academic Press, New York, 1975, pp. 191-224.

[6] The Theory of Partitions, Encyclopedia of Mathematics and Its Applications, Vol. 2, Addison-Wesley, 1976.

[7] Connection coefficient problems and partitions, Relations Between Combinatorics and Other Parts of Mathematics, D. K. Ray-Chaudhuri ed., Proc. Symp. Pure Math., 34 (1979), 1-24.

[8] __ Partitions and Durfee dissection, Amer. J. Math., 101 (1979), 735-742.

[9] _ The hard-hexagon model and Rogers-Ramanujan type identities, Proc. Nat. Acad. Sci. U.S.A., 78 (1981), 5290-5292.

[10] On the Wall polynomials and the $L-M-W$ conjectures, J. Austral. Math. Soc. (to appear).

[11] G. E. Andrews and R. Askey, Enumeration of partitions: the role of Eulerian series and q-orthogonal polynomials, from Higher Combinatorics, M. Aigner ed., Reidel, Dordrecht, 1977, pp. 3-26.

[12] W. N. Bailey, Some identities in combinatory analysis, Proc. London Math. Soc., (2), 49 (1947), 421-435.

[13] _ Identities of the Rogers-Ramanujan type, Proc. London Math. Soc., (2), 50 (1949), 1-10.

[14] D. M. Bressoud, Analytic and combinatorial generalizations of the Rogers-Ramanujan identities, Memoirs Amer. Math. Soc., 24 (1980), No. 227, 54 pp.

[15] _ _ Some identities for terminating q-series, Math. Proc. Camb. Phil. Soc., 89 (1981), 211-223.

[16] F. H. Jackson, Examples of a generalization of Euler's transformation for power series, Messenger of Math., 57 (1928), 169-187.

[17] S. Milne, A generalization of Andrews' reduction formula for the Rogers-Selberg functions, Amer. J. Math., 104 (1982), 635-643.

[18] L. J. Rogers, Second memoir on the expansion of certain infinite products, Proc. London Math. Soc., 25 (1894), 318-343.

[19] _ On two theorems of combinatory analysis and some allied identities, Proc. London Math. Soc., (2), 16 (1917), 315-336.

[20] L. J. Rogers and S. Ramanujan, Proof of certain identities in combinatory analysis, Proc. Camb. Phil. Soc., 19 (1919), 211-214. 
[21] I. Schur, Ein Beitrag zur additiven Zahlentheorie und zur Theorie der Kettenbrüche, Sitz.-Ber. Preuss. Akad. Wiss. Phys.-Math. Kl., 1917, pp. 302-321. (Reprinted in I. Schur, Gesammelte Abhandlungen, Vol. 2, pp. 117-136, Springer, Berlin, 1973).

[22] L. J. Slater, A new proof of Rogers's transformations of infinite series, Proc. London Math. Soc. (2), 53 (1951), 460-475.

[23] _ Further identities of the Rogers-Ramanujan type, Proc. London Math. Soc. (2), 54 (1952), 147-167.

[24] Generalized Hypergeometric Functions, Cambridge University Press, London and New York, 1966.

[25] A. Verma and V. K. Jain, Transformations between basic hypergeometric series on different bases and identities of Rogers-Ramanujan type, J. Math. Analysis and Appl., 76 (1980), 230-269.

[26] - Transformations of non-terminating basic hypergeometric series, their contour integrals and applications to Rogers-Ramanujan identities, J. Math. Analysis and Appl., 87 (1982), 9-44.

[27] G. E. Wall, On the conjugacy classes in the unitary, symplectic and orthogonal groups, J. Australian Math. Soc., 3 (1963), 1-62.

[28] G. N. Watson, A new proof of the Rogers-Ramanujan identities, J. London Math. Soc., 4 (1929), 4-9.

Received October 27, 1982 and in revised form January 4, 1983. Partially supported by National Science Foundation Grant MCS 8201733, and by a Guggenheim Fellowship.

The Pennsylvania State University

UNIVERSITY PARK, PA 16802 



\section{PACIFIC JOURNAL OF MATHEMATICS EDITORS}

\author{
DONALD BABBITT (Managing Editor) \\ University of California \\ Los Angeles, CA 90024 \\ J. DugundJI \\ University of Southern California \\ Los Angeles, CA 90089-1113 \\ R. FINN \\ Stanford University \\ Stanford, CA 94305 \\ HERMANN FLASChKa \\ University of Arizona \\ Tucson, AZ 85721
}

C. C. MOORE

University of California

Berkeley, CA 94720

Arthur Ogus

University of California

Berkeley, CA 94720

Hugo RossI

University of Utah

Salt Lake City, UT 84112

H. SAMELSON

Stanford University

Stanford, CA 94305

ASSOCIATE EDITORS
R. ARENS
E. F. BECKENBACH
B. H. NeUmanN
F. WOLF
K. YosHIDA (1906-1982)

\section{SUPPORTING INSTITUTIONS}
UNIVERSITY OF ARIZONA
UNIVERSITY OF BRITISH COLUMBIA
CALIFORNIA INSTITUTE OF TECHNOLOGY
UNIVERSITY OF CALIFORNIA
MONTANA STATE UNIVERSITY
UNIVERSITY OF NEVADA, RENO
NEW MEXICO STATE UNIVERSITY
OREGON STATE UNIVERSITY

\author{
UNIVERSITY OF OREGON \\ UNIVERSITY OF SOUTHERN CALIFORNIA \\ STANFORD UNIVERSITY \\ UNIVERSITY OF HAWAII \\ UNIVERSITY OF TOKYO \\ UNIVERSITY OF UTAH \\ WASHINGTON STATE UNIVERSITY \\ UNIVERSITY OF WASHINGTON
}

The Supporting Institutions listed above contribute to the cost of publication of this Journal, but they are not owners or publishers and have no responsibility for its content or policies.

Mathematical papers intended for publication in the Pacific Journal of Mathematics should be in typed form or offset-reproduced (not dittoed), double spaced with large margins. Please do not use built up fractions in the text of the manuscript. However, you may use them in the displayed equations. Underline Greek letters in red, German in green, and script in blue. The first paragraph must be capable of being used separately as a synopsis of the entire paper. In particular it should contain no bibliographic references. Please propose a heading for the odd numbered pages of less than 35 characters. Manuscripts, in triplicate, may be sent to any one of the editors. Please classify according to the scheme of Math. Reviews, Index to Vol. 39. Supply name and address of author to whom proofs should be sent. All other communications should be addressed to the managing editor, or Elaine Barth, University of California, Los Angeles, California 90024.

There are page-charges associated with articles appearing in the Pacific Journal of Mathematics. These charges are expected to be paid by the author's University, Government Agency or Company. If the author or authors do not have access to such Institutional support these charges are waived. Single authors will receive 50 free reprints; joint authors will receive a total of 100 free reprints. Additional copies may be obtained at cost in multiples of 50 .

The Pacific Journal of Mathematics is issued monthly as of January 1966. Regular subscription rate: $\$ 190.00$ a year (5 Vols., 10 issues). Special rate: $\$ 66.00$ a year to individual members of supporting institutions.

Subscriptions, orders for numbers issued in the last three calendar years, and changes of address should be sent to Pacific Journal of Mathematics, P.O. Box 969, Carmel Valley, CA 93924, U.S.A. Old back numbers obtainable from Kraus Periodicals Co., Route 100, Millwood, NY 10546.

The Pacific Journal of Mathematics at P.O. Box 969, Carmel Valley, CA 93924 (ISSN 0030-8730) publishes 5 volumes per year. Application to mail at Second-class postage rates is pending at Carmel Valley, California, and additional mailing offices. Postmaster: Send address changes to Pacific Journal of Mathematics, P.O. Box 969, Carmel Valley, CA 93924.

PUBLISHED BY PACIFIC JOURNAL OF MATHEMATICS, A NON-PROFIT CORPORATION

Copyright $\odot 1984$ by Pacific Journal of Mathematics 


\section{Pacific Journal of Mathematics}

Vol. 114, No. $2 \quad$ June, 1984

William Allen Adkins, A Harnack estimate for real normal surface

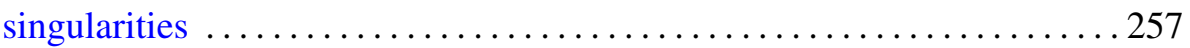

George E. Andrews, Multiple series Rogers-Ramanujan type identities . . . . 267

Didier Arnal, $*$ products and representations of nilpotent groups . . . . . . 285

David Cox and Walter Raymond Parry, Representations associated with

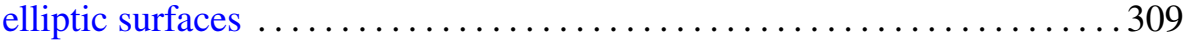

Joanne Marie Dombrowski, Tridiagonal matrix representations of cyclic

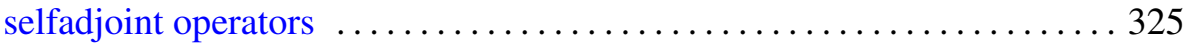

Ronald Dotzel, An Artin relation $(\bmod 2)$ for finite group actions on

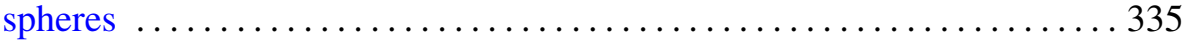

Leo Egghe, Convergence of adapted sequences of Pettis-integrable

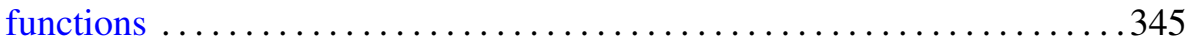

Rebecca A. Herb, Characters of induced representations and weighted

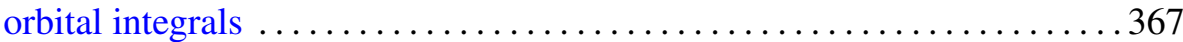

Steven M. Kahn, Cobordism obstructions to fibering manifolds over

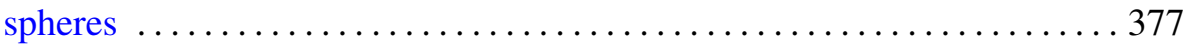

Robert D. Little, Projective space as a branched covering of the sphere with orientable branch set

Claude Schochet, Topological methods for $C^{*}$-algebras. III. Axiomatic

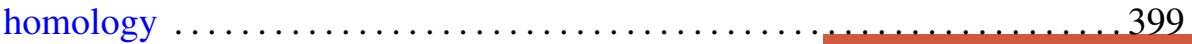

Claude Schochet, Topological methods for $C^{*}$-algebras. IV. $\bmod p$ homology

James M. Stormes, On the $K O$-orientability of complex projective varieties

Josephine Anne Ward, Characterization of homogeneous spaces and their norms 